\title{
Adaptation of Polystyrene/Multi-Wall Carbon Nanotube Composite Properties In Respect of its Thermal Stability
}

\author{
Petr Slobodian, ${ }^{1}$ Daniela Králová, ${ }^{2}$ Anežka Lengálová, ${ }^{1}$ Radko Novotný, ${ }^{3}$ Petr Sáha'
}

\begin{abstract}
Polystyrene (PS)/multi-wall carbon nanotube (MWCNT) nanocomposites were prepared by melt mixing and by coagulation of PS/MWCNT sonicated dispersion. Before mixing and coagulation particles of MWCNT were covered with PS nanospheres by microemulsion polymerization to increase their dispersibility and compatibility with the matrix. Microemulsion polymerization was carried out under sonication in the presence of surfactants and a cross-linking agent. The structure of modified MWCNT filler was revealed by SEM and TEM microscopy analyses. They show that PS is well grafted onto surface of MWCNT in form of ca $30 \mathrm{~nm}$ nanospheres or as ca 3-8 nm PS film layer. Significant changes in properties were recorded mainly for the coagulated sample. Although no shift of $T_{g}$ was recorded for the melt mixed composite, the coagulated sample shows an increase by $9^{\circ} \mathrm{C}$. Also the reinforcing effect of MWCNT was more pronounced for the same method of preparation (further supported by the filler modification). The creep response of this material shifts to longer times not only in the $T_{g}$ area, but also as low as $70 \mathrm{C}$ below it, which is perceived as an improvement of temperature stability (the same properties kept for a longer time). This was also proved by the increase of creep modulus, and consequently by the prolonged time needed to reach the same tensile creep compliance after aging. POLYM. COMPOS., 31:452- 458, 2010. ( 2009 Society of Plastics Engineers
\end{abstract}

\section{INTRODUCTION}

Polymer/carbon nanotube (CNT) composites are a type of highly promising materials, where specific properties of both principal types of CNT-MWCNT (multi-wall) and SWCNT (single-wall)—can be effectively availed. The nanoparticles are incorporated into a matrix, which gives a rise of a new multifunctional material with enhanced mechanical, thermal, or electrical properties [1], Practically one-dimensional CNT with high flexibility and an aspect ratio close to $10^{\prime \wedge}$ give rise to elastic modulus of

0. 3-1 TPa for MWCNT and ca $1 \mathrm{TPa}$ for SWCNT, or mechanical strength of 10-60 GPa and 50-500 GPa, respectively. This unique polymer reinforcement enables very specific application of the materials [2,3]. Furthermore, relatively high improvement of mechanical properties is provided at the level of filling in only units of wt $\%$. For instance, polypropylene/SWCNT system produced an increase in Young's modulus from $0.85 \mathrm{GPa}$ for pure matrix to $1.19 \mathrm{GPa}$ for $0.75 \mathrm{wt} \%$-filled nanocomposite [4], or for polyethylene/SWNCT composite the tensile strength and initial modulus rose by about $60 \%$ and $50 \%$, respectively, at CNT loading level of only $2.6 \mathrm{wt} \%$ [5]. Another example of significantly improved properties (increase in Young's modulus by about 27\%) and, on the other hand, decrease of elongation at break was reported for PA6 matrix [6J.

Unfortunately, there are still significant difficulties accompanying fabrication of this type of composites. Among others, it is very hard to distribute nanotubes uniformly throughout polymer matrix as they are strongly attracted to each other by van der Waals forces. Thus, it is very difficult to deagglomerate the bundles or ropes (in the case of MWCNT [7] or SWCNT [8], respectively) using conventional methods of melt processing. Practically, in the nanocomposites prepared by convectional 
melt mixing methods agglomerates of nanotubes of micrometric size or larger can be observed in the matrix, e.g. PP [4], poly (methyl methacrylate) (PMMA) [9], high-den- sity poly (ethylene) (HDPE) [5, 10, 11], or PA6 [6]. The aggregates of CNT considerably reduce the aspect ratio of the tubes, and thus the reinforcing effect [4]. Also load transfer can be limited because of the bundles of nanotubes; when some of the tubes are not bonded to the matrix, they slip within the bundles, and it is easier for them to slide out of the bundle than to break [12, 13].

Another possibility how to fabricate polymer nanocomposites is solvent-based methods [9, 14-16]. Better CNT distribution can be reached by, for example, coagulation of CNT dispersion in polymer solution by its dropping into stirred antisolvent [14-16]. The PMMA/SWCNT composite prepared by this method with amide functional- ized SWCNT shows uniform filler dispersion.

Incorporation of filler can cause changes in properties. For instance, a really high increase in glass transition temperature of PMMA was found after filling (from $105^{\circ} \mathrm{C}$ for pure material to $138^{\circ} \mathrm{C}$ for composite) although usually little or no $T_{g}$ changes are reported [17]. In context of [15], the question is only whether this significant increase in $T_{g}$ is really caused by reduced molecular mobility in the polymer-CNT interface because of well-distributed filler or by effective cross-linking of the tubes in the polymer network because of the method of composite preparation. In another system prepared by coagulation [16], the addition of less than $0.1 \mathrm{wt} \%$ SWCNT to PMMA led to an increase in the low-temperature elastic modulus by $\wedge 10 \%$ compared with pure PMMA matrix, without any change in the value of $7 \mathrm{~g}$ and modulus measured in glass transition region. Another PMMA/SWCNT composite showed an increase of elastic modulus by about $90 \%$ for $2 \mathrm{wt} \%$ loading [14]. In all cases, the effect of the nanotubes exceeded the prediction based on theoretical models. The above discussed coagulation method can be practically applied to a continuous operation in large-scale production and furthermore, it is applicable to a wide range of thermoplastics, providing that an appropriate combination of solvents is used [14].

Another major problem in the area of polymer/CNT nanocomposites is the bonding level between the filler and polymer matrix [2]. At present, there are no reliable rules for predicting compatibility between CNT and polymer matrix, which would prevent from poor load transfer across the nanotubes/polymer interface [1, 2, 13]. Therefore, it is very difficult to control key mechanical properties of the composite, such as elastic modulus, tensile strength, fracture toughness, or long-term behavior [18], even if relatively good adherence of polymer matrices to CNT surface has also been reported [18-20], for instance PS/CNT composite shows good wetting of the tube surface by the polymer, together with strong matrix-filler adhesion.

One potential way is how to prepare high-performance polymer/CNT composites with better filler distribution and compatibility can be chemical or chemical-physical modification of the filler by the method of covalent or noncovalent CNT functionalization. An example of the first one can be CNT and polymer in situ polymerization, which is often used to ensure chemical bonding between the two components, and thus, to improve the composite properties [21, 22]. A noncovalent method was shown in [23-25], where polymeric material in the form of nanospheres was adsorbed onto the surface of CNT through microemulsion polymerization technique. Also here the aim was the same-better dispersion of the filler and stronger CNT/polymer interface.

The supposed mechanism of microemulsion polymerization in the presence of CNT has been described by Ham et al. [23]. In this case, the method was oil-in-water free radical type of microemulsion polymerization stabilized by an anionic surfactant (sodium dodecyl sulfate) and a co-surfactant (1-penthanol). The prepared PS/ SWCNT composite contains a large proportion of carbon nanotubes covered with polystyrene (PS) nanospheres with a diameter of $\sim 30 \mathrm{~nm}$. Some parts of nanotubes are covered with thin PS film and others are without PS coating. The PS/SWCNT composite prepared by microemulsion polymerization was measured for storage modulus by dynamic mechanical analysis and showed a significant increase compared with pure PS. However, CNT content was $13.1 \mathrm{wt} \%$, which is very high for a nanofiller.

The submitted paper deals with the procedure of PS/ MWCNT composite preparation, which was optimized step by step until a significant improvement in physical properties was reached with a minimum amount of CNT filler. Individual steps of composite fabrication were gradually developed: from the use of unmodified and modified MWCNT, through melt mixing and using composite master batch to final coagulation of polymer/filler dispersion. In the present research, we tested an application of a relatively cheap and simple-to-use method. An inexpensive unmodified commercial type of CNT filler was used for PS/MWCNT nanocomposite preparation by microemulsion polymerization under ultrasound. Nanotube-polystyrene-grafted nanocomposite (MWCNT-g-PS) was polymerized and then melt mixed in PS matrix in total CNT concentration of $0.5 \mathrm{wt} \%$. Finally, another composite was prepared by precipitation of melt mixed composite solution in methylethyl ketone (MEK) in water. The efficiency of CNT reinforcement was assessed mainly via tensile creep tests and the results were compared with pure PS matrix and discussed from the standpoint of composite thermal stability.

\section{EXPERIMENTAL}

\section{Materials}

The polymer matrix used was commercial PS (Krasten 137, Kaucuk-Unipetrol Group, $\left.M_{n}=102530, \mathrm{M}_{\mathrm{w}} / \mathrm{M}_{\mathrm{n}}=2.75\right)$. The filler was multi-wall carbon nanotubes 
(MWCNT), acetylene type, purified, by Sun Nanotech Co. Ltd., China (diameter 10-30 nm, length $1-10 / \mathrm{im}$, purity >90\%). Styrene as a monomer, divinylbenzene as a cross-linking agent (inhibitors alkaline removed), sodium dodecyl sulfate and 1pent.anol as surfactants, sodium bicarbonate as a buffer reagent, $2,2^{\prime}$-azobisisobutyronitrile as a free radical initiator, toluene and MEK as solvents of PS were used.

\section{Microemulsion Polymerization}

Sodium dodecyl sulfate $(27.5 \mathrm{~g})$, 1-pentanol $\left(15 \mathrm{~cm}^{3}\right)$, and sodium bicarbonate $(2.1 \mathrm{~g})$ were dissolved in $250 \mathrm{~cm}^{3}$ of deionized water. Then $0.9 \mathrm{~g}$ MWCNT was added and dispersed with the help of ultrasound (Hielscher GmbH, UP 400s apparatus with ultrasonic horn S7) for 120 min under nitrogen atmosphere.

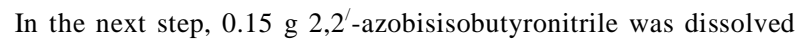
in styrene $\left(15 \mathrm{~cm}^{3}\right)$ mixed with divinylbenzene $\left(1.6 \mathrm{~cm}^{\mathrm{J}}\right)$ and charged together with the prepared MWCNT dispersion into a $500 \mathrm{~cm}^{3}$ glass reactor fitted with a condenser and nitrogen inlet. The temperature of the reactor was raised to $85{ }^{\circ} \mathrm{C}$ to initiate polymerization for $4 \mathrm{hr}$ under ultrasound in nitrogen atmosphere. The temperature was controlled by thermostatic ultrasonic bath (Bandelin electronic DT 103H). The resulting emulsion was centrifuged at $9000 \mathrm{rpm}$ for $4 \mathrm{hr}$. The white part containing mainly PS particles was decanted; the black-colored supernatant with MWCNT-g-PS particles was washed with ethanol and water and dried.

\section{Nanocomposites}

Two different methods of bulk nanocomposites preparation were used. The first was classical melt mixing of MWCNT-g-PS or MWCNT with PS in a laboratory compounder and the other was coagulation of PS solution in MEK where filer was sonically dispersed. Here, in the first step master batches from PS Krasten and MWCNT-g-PS or MWCNT were prepared as $15 \mathrm{wt} \%$ PS solution in toluene, with the help of ultrasound for $10 \mathrm{~min}$; the total concentration of MWCNT was about $2.5 \mathrm{wt} \%$. These mixtures were cast into a form of film and dried. The master batches were the basis for the preparation of melt mixed nanocomposite using nontreated, as delivered MWCNT (samples further denoted as PS/MWCNT) and (PS/MWCNT-g-PS) mix, using CNT filler covered with PS nanospheres. The process of mixing was carried out in micro compounder HAAKETM with a co-rotating twin- screw for $20 \mathrm{~min}$, at the barrel temperature of $170^{\circ} \mathrm{C}$ and the screw speed of $30 \mathrm{rpm}$ The final filler concentration was about $0.5 \mathrm{wt} \%$. In the second type of preparation, coagulation method, the mixed composite (PS/MWCNT- g-PS) $)_{\text {mix }}$ was dissolved in MEK to create $15 \mathrm{wt} \%$ PS solution and sonicated for $30 \mathrm{~min}$. Then the dispersion was poured into excess amount of room-temperature water under stirring. Coagulated (PS/MWCNT-g-PS) $)_{\text {coag. }}$. nanocomposite was filtered and dried at $125^{\circ} \mathrm{C}$ under vacuum.

\section{Analyses}

The structure of composites containing nanotubes in the original and modified forms, MWCNT and MWCNT- g-PS, respectively, was analyzed by scanning electron microscopy (SEM). The studied materials were deposited onto carbon targets and covered with 4-nm platinum layer using sputter coater SCD050, BAL-TEC. The specimens were observed in the regime of secondary electrons in microscope Quanta 200 FEG under high vacuum. Finally, the TEM analyses of MWCNT-g-PS were performed using the transmission electron microscope JEOL JEM 2010 at the accelerating voltage $160 \mathrm{kV}$. Samples for TEM were prepared using 300 mesh cooper grids with holey carbon film (SPI, USA). Thermogravimetric behavior of the samples was studied using thermogravimeter SETARAM SETSYS Evolution 1200. The samples were examined under an inert atmosphere of helium (5.5 purity, SIAD TP); the gas flow was $30 \mathrm{~cm}^{3} / \mathrm{min}$ at the pressure of $101.325 \mathrm{kPa}$ (i.e., 30 seem) for all experiments. A platinum crucible was used for the sample, the weight of which was about $4 \mathrm{mg}$. Temperature was increased at the rate of $20^{\circ} \mathrm{C} / \mathrm{min}$, in the range from the ambient temperature up to $1200^{\circ} \mathrm{C}$ for nonisothermal analysis. Differential scanning calorimetry, DSC (Perkin-Elmer DSC 1 Pyris), was used to measure glass transition temperature, $\mathrm{T}_{\mathrm{g}}$. The temperature and heat flow of the apparatus was calibrated on indium standard. The following temperature program was chosen: annealing at $155^{\circ} \mathrm{C}$ for 5 min cooling to $65^{\circ} \mathrm{C} \rightarrow$ reheating through $T_{g}$ region up to $125^{\circ} \mathrm{C}$ (cooling/heating rates $\pm 10^{\circ} \mathrm{C} / \mathrm{min}$ ). Tensile creep behavior was determined on a home-made laboratory device producing pure tension. The specimens were prepared by compression molding at $175^{\circ} \mathrm{C}$, and their shape and dimensions were according to standard EN ISO 3167. Before each measurement the specimen was annealed at $(\mathrm{Tg}+15)^{\circ} \mathrm{C}$ for $30 \mathrm{~min}$ between two iron plates; then it was cooled in room-temperature water. Time zero for aging was the moment of immersing the hot specimen into cooling water. At an appropriate aging time, $r_{a}$, the sample was subjected to a constant stress, $c r_{0}$, and the resulting strain, $£$, was measured as a function of elapsed creep time, $t$ (begins at the moment of loading). Principally, two types of creep experiments were performed and assessed as (1) a change of compliance with the time elapsed from the temperature jump (influence of physical aging) (2) an effect of different temperatures on the creep. Finally, tensile creep tests were performed at heating under constant rate.

\section{RESULTS AND DISCUSSION}

The structures of as-received nanotubes, MWCNT, and the product of microemulsion polymerization, MWCNT- 

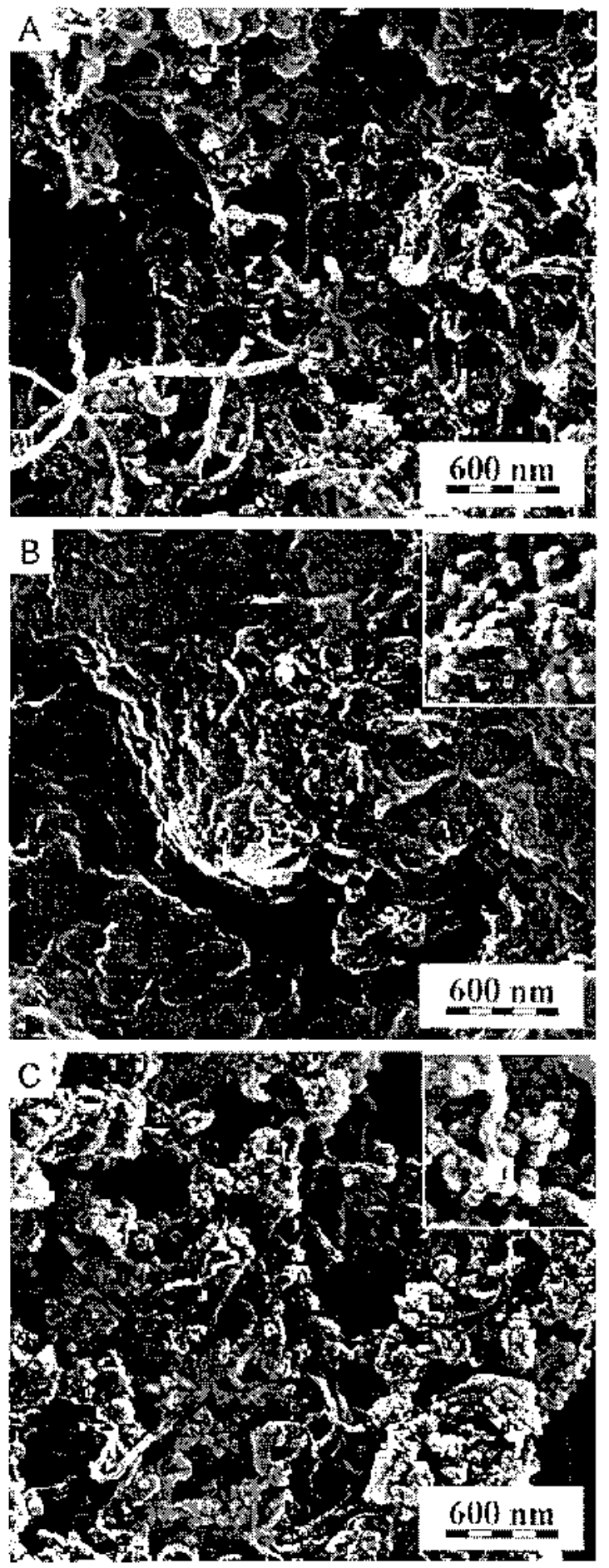

FIG. 1. SEM analysis: A-Original MWCNT, B-MWCNT-g-PS nanocomposite, (inset $2.5 \mathrm{X}$ magnified), $\mathrm{C}$-sonicated MWCNT-g-PS composite in methanol (inset $2.5 \mathrm{X}$ magnified).

g-PS, nanocomposites were examined by SEM microscopy, and the pictures are presented in Fig. 1. Figure 1A represents MWCNT in the form delivered by the supplier. Here, the differences in diameter of individual nanotubes can be observed; the size varies from ca 10 to $60 \mathrm{~nm}$ (different from the producer specification). Also particles of different shape, which is probably carbon black, are apparent. Figure IB and C represents the product of microemulsion polymerization in different stages of preparation. Figure IB represents MWCNT-gPS particles after drying. The tubes are covered with core created by PS nanospheres (see the figure intersection). Figure $1 \mathrm{C}$ represents the structure of MWCNT-g-PS nanocomposite after sonication in liquid. PS nanospheres adsorbed on the surface of nanotubes can be observed, but some of the tubes seem to be without PS coverage. As can be seen, the diameter of the beads (cross-linked PS nanospheres prepared during polymerization) is about $30 \mathrm{~nm}$. The cross-linking was assured by divinylbenzene, and its purpose was to avoid possible PS elution from the tubes (i.e., complete extraction by benzene [23]) in the following steps in PS matrix melt or solution. Additional TEM analyses shows good adherence of PS beads onto MWCNT surface, Fig. 2A, specimen prepared by sonication in PS solvent (acetone). Finally, the presence of polymer film layer adhering onto the MWCNT surface was revealed, indicated by arrow (1). Its detailed structure is presented in Fig. 2B. The image shows one wall of MWCNT tube assembled from $\wedge 25$ of individual graphitic layers with distance between particular layer $\sim 0.35 \mathrm{~nm}$ covered by grafted PS layer with thickness of $\sim 3.5 \mathrm{~nm}$. Further analyses of MWCNT-gPS nanocomposite proved overall coverage of MWCNT surface by PS with average thickness varying between 3 and $8 \mathrm{~nm}$. This PS layer would be partially covalently bonded to the MWCNT surface as was experimentally demonstrated in [21, 26] where sonochemical bulk polymerization of styrene in MWCNT presence leads to open 71-bonds in the MWCNT wall.

The content of PS grafted onto the surface of MWCNT was determined with the help of thermogravimetric (TG) analyses. Figure $3 \mathrm{a}$ shows pure MWCNT as-received material, Fig. 3b represents the PS matrix, and Fig. 3c represents the MWCNT-gPS nanocomposite. As can be seen, MWNT shows hardly any degradation in the used range of heating, up to $700^{\circ} \mathrm{C}$; only a very small mass loss of ca $3 \%$ at the highest temperature can be noticed. On other hand, the main chain of PS (Fig. 3b) starts to decay at the temperature of about $400^{\circ} \mathrm{C}$ and is totally decomposed at some $460^{\circ} \mathrm{C}$. For MWCNT-g-PS nanocomposites the situation is slightly more complicated (Fig. 3c); two decomposition events are visible here. The first one is located at ca $220^{\circ} \mathrm{C}$ (15\% mass loss) and is probably caused by some lower PS fractions or rest of unwashed surfactants (the content of umvashed surfactants in MWCNT-g-PS nanocomposites was confirmed by FT-IT spectra). The decomposition at about $420^{\circ} \mathrm{C}$ corresponds to the main chain of PS deterioration $(57.5 \%$ mass loss) and is finished at a slightly higher temperature then for pure PS - at some $480^{\circ} \mathrm{C}$. The amount of MWNT in MWCNT-g-PS nanocomposite was determined as the difference in resid- 


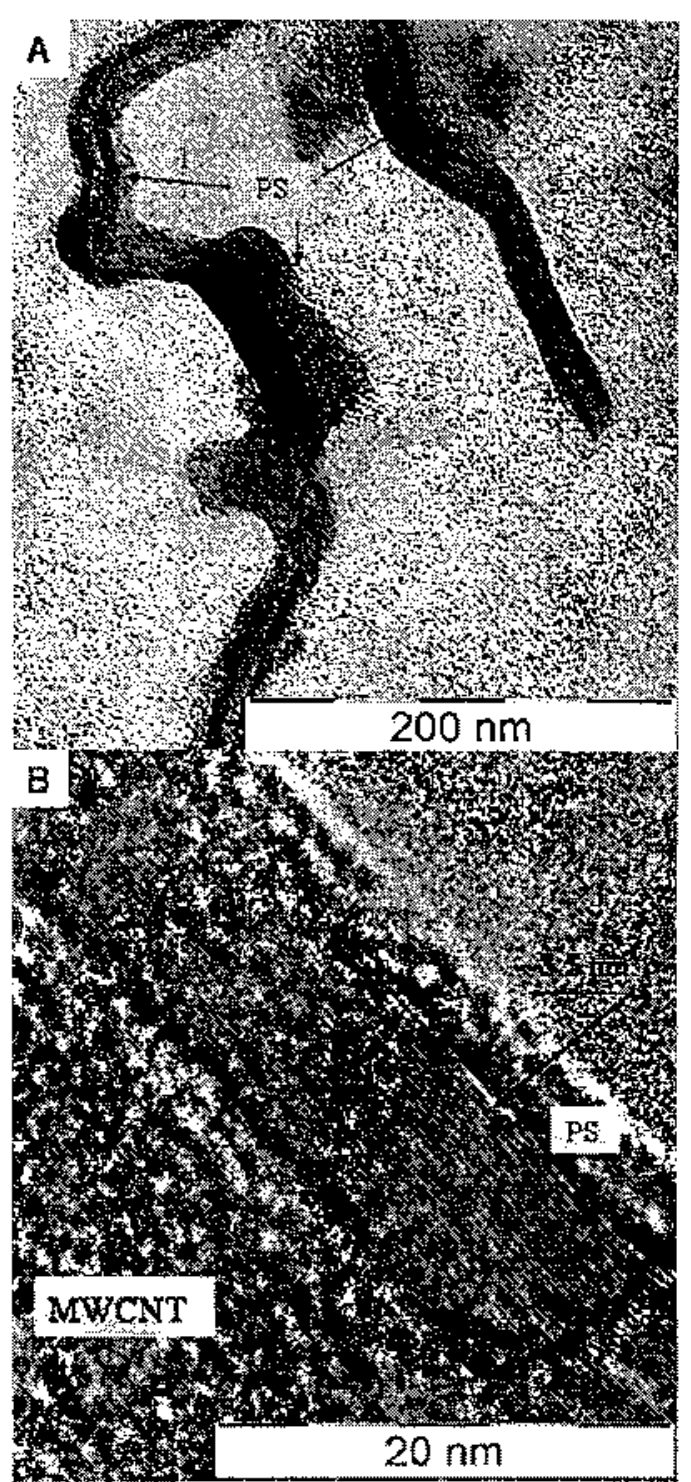

FIG. 2. TEM morphology of MWCNT-g-PS nanocomposite.

ual weight between PS, MWNT, and MWCNT-g-PS at $700^{\circ} \mathrm{C}$ with the resulting value of about $28.2 \mathrm{wt} \%$.

DSC analysis did not show any significant difference in thermal behavior of the prepared melt mixed composites and the used PS matrix (not presented here graphically). Glass transition temperature, $T_{g}$, of all three materials, PS, PS/MWCNT, and(PS/MWCNT-g-PS) mix., was measured to be around $92^{\circ} \mathrm{C}$ (evaluated as the peak temperature), with the enthalpy of $T_{g}$ transformation of about $1.0 \mathrm{~J} / \mathrm{g}$. On other hand, the sample prepared by coagulation (PS/MWCNT-g-PS) coag. shows a significant increase of $\mathrm{T}_{\mathrm{g}}$ reaching the value of $101^{\circ} \mathrm{C}$.

This increase in transformation temperature can be also demonstrated by a different type of experiment, tensile creep heating test, see Fig. 4. At lower temperatures the deformation is nearly the same for all the materials. At

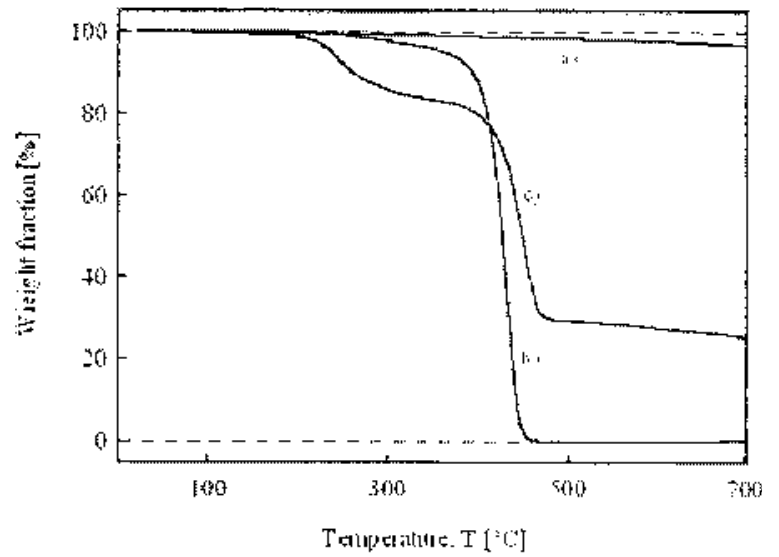

FIG. 3. Thermogravimetric analysis of as-received MWCNT (a), pure PS (b), and MWCNT-g-PS nanocomposite (c).

the upper limit of glass transition region the samples start to more significantly creep under the stress from loading. A slight shift caused by the presence of CNT material in melt mixed samples can be noticed, which is more pronounced for MWCNT-g-PS filler. This shift is, however, about $1.5^{\circ} \mathrm{C}$ to higher temperatures. A more significant increase, ca $9^{\circ} \mathrm{C}$, was measured for the coagulated sample, (PS/MWCNT-g-PS) $)_{\text {coag, }}$,

The main focus of the research, however, was on the creep behavior. It was measured under tensile load and assessed as tensile creep compliance, $J_{(t} y$.

$$
J(t)=\frac{\varepsilon(t)}{\sigma_{t 1}}-\frac{A_{t 1}[A L(t)]}{H_{L}}-\frac{1}{E(t)}
$$

where $\mathrm{e}(\mathrm{f})$ is the relative strain as a function of creep time $t$, (To means the initial stress, $A_{0}$ represents the initial cross section of the sample, $\mathrm{A} L(t)$ is the sample extension at

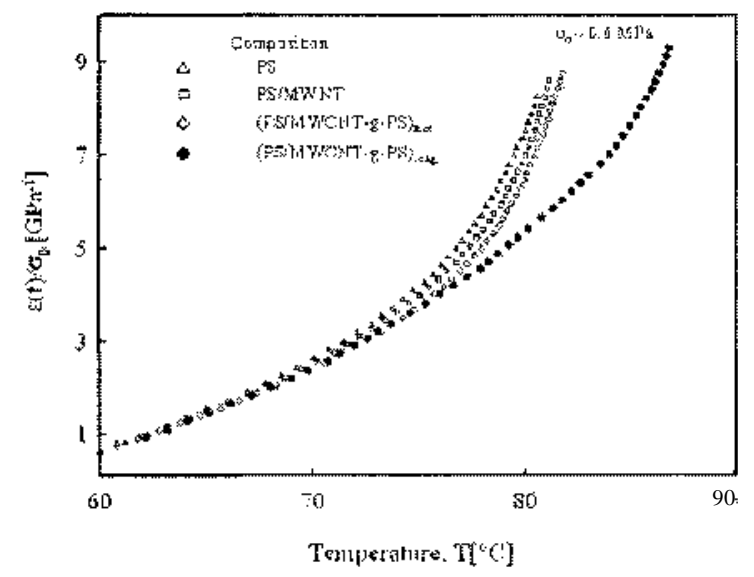

FIG. 4. Tensile creep heating curves for the prepared composites, constant heating rate $1^{\circ} \mathrm{C} / \mathrm{min}$, after temperature jump and aging at $60^{\circ} \mathrm{C}$ for $0.5 \mathrm{hr}$. The initial stress $<7_{0}-0.6 \mathrm{MPa}$. 


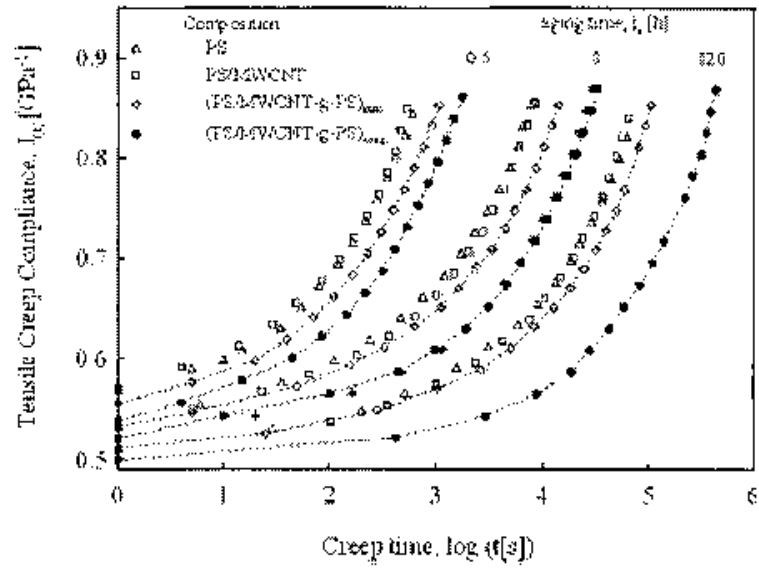

FIG. 5. Tensile creep curves for pure PS, PS/MWCNT, (PS/MWCNT- g-PS) $)_{m} \mathrm{j}_{\mathrm{X}}$, and (PS/MWCNT-g-PS) $)_{\text {coa }} \mathrm{g}$ composites after temperature jump and aging times $0.5,8$, and $120 \mathrm{hr}$. The crosses in the curve for $8 \mathrm{hr}$ prove good reproducibility.

time $t, F$ means the extension force, $\mathrm{L}_{0}$ stands for initial effective length of the sample, and $E(t)$ is tensile creep modulus reciprocal to $J(t)$.

The influence of carbon nanotubes onto tensile creep properties is demonstrated in Fig. 5; all four materials are compared for examples of three aging times $(0.5,8$, and $120 \mathrm{hr}$ ) relaxed at $\mathrm{T}_{\mathrm{a}}=60^{\circ} \mathrm{C}$. The crosses for $8 \mathrm{hr}$ demonstrate reproducibility of the test method. It seems that the presence of neat MWCNT (0.5 wt.\%) does not affect the tensile compliance of PS matrix - the curves are nearly identical. On the other hand, using the filler modified by microemulsion polymerization reflects in shifting the curve position horizontally along the time axis, which means that the structure of the nanocomposite is more rigid. In other words, the same deformation of the material is achieved in a longer time. The horizontal shift factor was calculated to be nearly the same for different elapsed times; its value is ca $0.2 \mathrm{dec} \log$. A much more significant shift was found for coagulated material. This effect is more pronounced by both elapsed aging time, $\mathrm{f}_{\mathrm{a}}$, and creep time, $t$. For the experimental conditions with the longest time, it means aging at $60^{\circ} \mathrm{C}$ for 120 $\mathrm{hr}$, the same compliance $J(t)=0.85 \mathrm{GPa} \sim{ }^{1}$ is achieved for pure PS matrix after $29 \mathrm{hr}$ as for (PS/MWCNT-g-PS) $)_{\text {coag. after nearly }}$ $110 \mathrm{hr}$. Thus the material stiffness improvement can be estimated as some $380 \%$ increase in creep resistance of the sample at the defined experimental conditions.

The figure shows how the curves for higher $i_{a}$ are shifted along the time axis. This characteristic behavior reflects continuous solidification of the sample in the process of physical aging, which leads among others to continuous increase of the time of response to the deformation, i.e., shift of compliance curves to longer times [27, 28]. The same process also reduces the initial values of $/($,$) in different t_{a}$ and keeps them longer on lower values.
From this, double-logarithmic shift rate, //., was calculated for all four materials according to $E q$. 2:

$$
d-\cdots-\frac{d \log a}{d \log t}
$$

where $a$ represents the horizontal shift factor, $t$ is the creep time. However, no significant difference in /.( was found between pure PS and both melt mixed composites. They were: for PS $i J$, 0.82 , for PS/MWCNT $/ \mathrm{i}=0.83$, and for $(\mathrm{PS} / \mathrm{MWCNT}-\mathrm{g}-\mathrm{PS})_{\mathrm{m}} \mathrm{j}_{\mathrm{x}}, \mathrm{fi}$ - 0.81. On the other hand, a significant difference was found for the coagulated specimen (PS/MWCNT-g-PS) $)_{\text {coag. }}$ : $\mathrm{A}^{*}=0.98$. Struik [29] shows that i $x$ for many glass-formers reach the value of about unity in a broad range of $t$ at the temperature not far below $T_{g}$. For PS in the temperature range of $50-70^{\circ} \mathrm{C}$ the value of about 0.96 can be found [27]. For temperatures far below $T_{g}$ the aging process slows down, which leads to a decrease in $/ i$. The shift rate also decreases close to thermodynamic equilibrium, which is for reasonable experimental times only the case of creep temperatures within glass transition region. Here eventually the rate decreases to zero (at $T_{g}$ ). Thus, the higher value of shift rate for (PS/MWCNT-g-PS) $)_{\text {coag, }}$ in our case is most probably caused by the shift of $T_{g}$ of the composite to higher temperatures.

The data served to express the dependence of tensile creep modulus on temperature, Fig. 6. These moduli were determined for the set conditions of the experiment (randomly chosen) values of $E f t$ ) after $10^{3} \mathrm{~s}$ from the application of the load on the sample relaxed for $0.5 \mathrm{hr}$ at room temperature, and for $10^{4} \mathrm{~s}$ - the sample relaxed for $24 \mathrm{hr}$ at the same temperature. The moduli for both materials gradually decrease with increasing temperature, reaching very small values in $T_{g}$ area. It is logical that for PS, which has a lower $T_{g}$, this decrease appears sooner

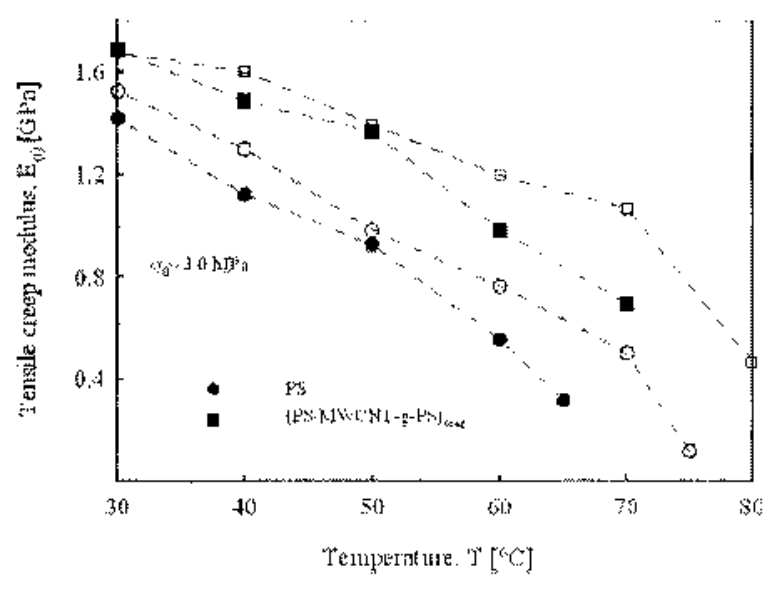

FIG. 6. Dependences of tensile creep moduli, $E(t)$, on the temperature of creep

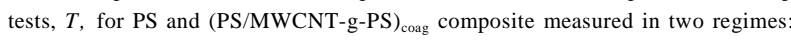
full symbols-after temperature jump to room temperature and aging for $24 \mathrm{hr}$, creep time $10^{4} \mathrm{~s}$; open symbols-after the same temperature jump and aging for $0.5 \mathrm{hr}$, creep time $10^{\mathrm{J}} \mathrm{s}$. The initial stress ( $7 Q$ for both type of tests $\sim 3.0 \mathrm{MPa}$. 
than for (PS/MWCNT-g-PS) coag. The graph, however, shows the shift of $E f t$ ) for the coagulated composite, compared with pure $\mathrm{PS}$, in the whole tested temperature range-from room temperature to $T_{g}$, i.e., even some $60-70^{\circ} \mathrm{C}$ below $T_{g}$. This difference further increases with approaching to $7 \mathrm{~g}$.

For the experiment with aging for $24 \mathrm{hr}$ at room temperature the values of creep modulus show an increase by $19 \%$ at $30^{\circ} \mathrm{C}$, $32 \%$ at $40^{\circ} \mathrm{C}, 48 \%$ at $50^{\circ} \mathrm{C}$, and finally $77 \%$ at $60^{\circ} \mathrm{C}$. The materia] reinforced by CNT reached better temperature stability not only in the $T_{g}$ area, but also in the whole tested temperature range, as it shows an increased creep resistance, even in a deep glassy state.

\section{CONCLUSION}

The research has shown a feasible way of preparation of polymer/MWCNT composite with the help of microemulsion polymerization. The quality of the product prepared by this method of noncovalent CNT functionalization was assessed with the help of SEM and TEM microscopy and TG analysis. It was shown that PS beads $(\sim 30 \mathrm{~nm})$ are adsorbed onto the surface of carbon nanotubes. Further TEM analyses reveal film PS coverage of MWCNT surface with thickness of PS layer around 3-8 nm.

The composites were prepared by two fundamentally different methods: melt mixing and solvent-based technique supported by ultrasound as a precipitation method producing composite floccules. The obtained materials were tested for thermal properties, namely $\mathrm{T}_{\mathrm{g}}$, with the result that, the appropriately coagulated sample shows apparently higher glass transition temperature (by $9^{\circ} \mathrm{C}$ ) compared with pure PS matrix or meltmixed composites.

The study of creep behavior of the materials proved the positive effect of the filler on thermal stability (in the meaning of keeping resistance to creep in a wider temperature range). This improvement is apparent not only in the $T_{g}$ area, as could be expected from the shift of $T_{g}$ to higher values at the coagulated sample, but also can be seen in the whole tested range as far as room temperature. However, this increase is more pronounced in the vicinity of $T_{g}$.

Another factor followed in the research was the effect of physical aging on stiffening of the composites. The highest increase was recorded for the coagulated sample, where the longest aging time produced $380 \%$ increase in the time when the same creep compliance was reached as for pure PS matrix.

The main contribution of the research can be considered the use of cheap commercial MWCNT and relatively easy modification of the particles, which results in increased thermal stability of the material in a broad temperature range from room temperature to glass temperature transformation defined as creep resistance.

\section{REFERENCES}

1. K. Schulte and A.H. Windle, Compos. Sci. TechnoL, 61, 111 (2007).
2. X.L. Xie, Y.W. Mai; and X.P. Yhou, Mater. Sei. Eng. R Rep., 49, 89 (2005).

3. J.P. Salvetat, A.J. Kulik, J.M. Bonard, G.A.D. Briggs, T. Stöckli, K. Metenier, S. Bonnamy, F. Beguin, N.A. Burnham, and L. Forro, Adv. Mater., 11, 161 (1999).

4. M.A.L. Manchado, L. Valentini, J. Biagiotti, and J.M. Kenny, Carbon, 43, 1499 (2005).

5. Q.H. Zhang, S. Rastogi, D.J. Chen, D. Lippits, and P.J. Lemstra, Carbon, 44, 778 (2006).

6. O. Meincke, D. Kaempfer, H. Weickmann, C. Friedrich, M. Vathauer, and H. Warth, Polymer, 45, 739 (2004).

7. H. Kathyayini, I. Willems, A. Fonseca, J.B. Nagy, and N. Nagaraju, Catal. Commun., 7(3), 140 (2006).

8. A. Thess, R. Lee, P. Nikolaev, H.J. Dai, P. Petit. J. Robert, C.H. Xu, Y.H. Lee, S.G. Kim, A.G. Rinzler, D.T. Colbert, G.E. Scuseria, D. Tománek, J.E. Fischer, and R.E. Smalley, Science, 273, 483 (1996).

9. P. Slobodian, A. Lengálová, M. Šlouf, and P. Sáha, J. Reinforced Plast. Compos., 26, 1705 (2007).

10. W.Z. Tang, M.H. San tare, and S.G. Advani, Carbon, 41, 2779 (2003).

11. T. McNally, P. Potschke, P. Halley, M. Murphy, D. Martin, S.E.J. Bell, G.P. Brennan, D. Bein, P. Lemoine, and J.P. Quinn, Polymer, 46, 8222 (2005).

12. P.M. Ajayan, L.S. Schadler, C. Giannaris, and A. Rubio, $A d v$. Mater., 12, 750 (2000).

13. L.S. Schadler, S.C. Giannaris, and P.M. Ajayan, Appl. Phys. Lett., 73, 3842 (1998).

14. F. Du, J.E Fischer, and K.1 Winey, ./. Polym. Sci. Part B: Polym. Phys., 41, 3333 (2003).

15. T. Ramanathan, IT. Liu, and L.C. Brinson, ./. Polym. Sci. Part B: Polym. Phys., 43, 3269 (2005).

16. K.W. Putz, C.A. Mitchell, R Krishnamoorti, and P.F. Green, /. Polym. Sci. Part B: Polym. Phys., 42, 2286 (2004).

17. F.M. Du, R.C. Scogna, W. Zhou, S Brand, J.E. Fischer, and K.1. Winey, Macromolecules, 37, 9048 (2004).

18. M. Wong, M. Paramsothy, X.J. Xu, Y. Ren, S. Li, and K. Liao, Polymer, 44, 7757 (2003).

19. H.D. Wagner, Chem. Phys. Lett., 361, 57 (2002).

20. C. Bower, R. Rosen, L. Jin, J. Han, and O. Zhou, Appl. Phys. Lett., 74, 3317 (1999).

21. S.T. Kim, H.J. Choi, and S.M. Hong, Colloid Polym. Sci., 285, 593 (2007).

22. J.U. Ha, M. Kim, J. Lee, S. Choe, I.W. Cheong, and S.E. Shim, J. Polym. Sci. Part A; Polym. Chem., 44, 6394 (2006).

23. H.T. Ham, Y.S. Choi, MG. Chee, and 1.J. Chung, J. Polym. Sci. Part A: Polym. Chem., 44, 573 (2006).

24. C.S. Chern and L.J. Wu, .I. Polym. Sci. Part ,4: Polym. Chem., 39, 3199 (2001).

25. H.T. Ham, Y.S. Choi, N. Jeong, and 1.J. Chung, Polymer, 46, 6308 (2005).

26. H.J. Choi, K. Zhang, and J.Y. Lim, ./. Nanosci. Nanotech-noL, 7, 3400 (2007).

27. L.C.E. Struik, Physical Aging in Amorphous Polymers and Other Materials, Elsevier, Amsterdam (1978). 28. J.M. Hutchinson, Prog. Polym. Sci., 20, 703 (1995). 